\title{
PAPER \\ Welch FFT Segment Size Selection Method for FFT Based Wide Band Spectrum Measurement*
}

\author{
Hiroki IWATA $^{\dagger \mathrm{a})}$, Student Member, Kenta UMEBAYASHI ${ }^{\dagger \mathrm{b})}$, Janne J. LEHTOMÄKI ${ }^{\dagger \dagger}$, \\ and Shusuke NARIEDA ${ }^{\dagger \dagger \dagger}$, Members
}

\begin{abstract}
SUMMARY We introduce a Welch FFT segment size selection method for FFT-based wide band spectrum measurement in the context of smart spectrum access (SSA), in which statistical spectrum usage information of primary users (PUs), such as duty cycle (DC), will be exploited by secondary users (SUs). Energy detectors (EDs) based on Welch FFT can detect the presence of PU signals in a broadband environment efficiently, and DC can be estimated properly if a Welch FFT segment size is set suitably. There is a trade-off between detection performance and frequency resolution in terms of the Welch FFT segment size. The optimum segment size depends on signal-to-noise ratio (SNR) which makes practical and optimum segment size setting difficult. For this issue, we previously proposed a segment size selection method employing a relationship between noise floor (NF) estimation output and the segment size without SNR information. It can achieve accurate spectrum awareness at the expense of relatively high computational complexity since it employs exhaustive search to select a proper segment size. In this paper, we propose a segment size selection method that offers reasonable spectrum awareness performance with low computational complexity since limited search is used. Numerical evaluations show that the proposed method can match the spectrum awareness performance of the conventional method with $70 \%$ lower complexity or less.

key words: cognitive radio, duty cycle, dynamic spectrum access, spectrum measurement, Welch FFT
\end{abstract}

\section{Introduction}

To resolve the spectrum scarcity problem, dynamic spectrum access (DSA), where secondary user (SU), which has lower priority to use the spectrum, can use the spectrum when primary user (PU) does not use the spectrum, is a promising approach [2]. In time domain DSA, spectrum sensing is a well-investigated key technique to recognize instantaneous state of the spectrum [3]. To realize DSA, spectrum sensing is required to achieve high accuracy, low latency and reasonably low cost, however it is difficult in

\section{Manuscript received February 22, 2017.}

Manuscript revised October 9, 2017.

Manuscript publicized January 18, 2018.

The authors are with Department of Electrical and Electronic Engineering, Tokyo University of Agriculture and Technology, Koganei-shi, 184-8588 Japan.

${ }^{\dagger}$ The author is with University of Oulu, P. O. BOX 4500 FIN90014, University of Oulu, Finland.

${ }^{\dagger \dagger}$ The author is with Department of Electrical and Computer Engineering, National Institute of Technology, Akashi College, Akashi-shi, 674-8501 Japan.

${ }^{*}$ This paper was presented in part at the IWSS at IEEE WCNC 2016, Qatar, Doha [1].

a)E-mail: iwa-hiro@st.go.tuat.ac.jp

b) E-mail: ume_k@cc.tuat.ac.jp (Corresponding author)

DOI: $10.1587 /$ transcom.2017EBP3069 practice to satisfy all the requirements.

Not only to resolve the issue of spectrum sensing but also to provide other benefits to DSA, advanced DSA, known as smart spectrum access (SSA), has been considered [4]-[6]. SSA is kind of DSA and SSA exploits useful prior information, such as statistical information of spectrum utilization by PUs (DC: duty cycle), to achieve efficient spectrum sharing smartly. It has been shown that using DC can enhance spectrum sensing performance [7]-[9]. It can also enhance spectrum management, channel selection, MAC protocol for DSA [10]-[12]. However, there exists a key issue that how we can obtain this information as accurately and efficiently as possible in SSA [4], [5].

For this issue, two-layer architecture for SSA was proposed, where the first layer corresponds to a DSA system consisting of PUs and SUs, and the second layer is a spectrum awareness system (SAS) [4]-[6]. For PU, any specific wireless system is not assumed, but general wireless system is assumed. On the other hand, the SAS is dedicated for spectrum measurements, estimation of statistical spectrum usage information such as duty cycle (DC) over wide band covering multiple various wireless systems, and provision of statistical spectrum usage information obtained by the measurements to SUs. Due to the two layers, DSA terminals no longer suffer from the high implementation cost as statistical information is provided by the SAS. On the other hand, two-layer SSA requires the other cost for implementations of SAS and communication between the SAS and the DSA system.

In this paper, we focus on SAS corresponding to second layer in SSA, especially spectrum measurements and estimation of statistical spectrum usage information. We focus on DC since it is useful statistical information. DC is defined as a probability that the channel is occupied where we regard the channel as one frequency bin in FFT [13]. DC has a potential to enhance spectrum sensing and channel selection in DSA [7], [10]. We assume that processes in the DSA system corresponding to the first layer in SSA such as spectrum sensing and spectrum sharing take the constraint for PU protection such as miss detection rate into account. However, we will investigate impact of errors of DC in spectrum sensing and spectrum sharing as our future work since the protection of PU is very important for DSA.

The assumed SAS consists of three functional blocks [5]. The first one is time-frequency domain conversion (such as FFT: fast Fourier transform) to obtain power spectrum 
density (PSD) on a time-frequency grid. The second one is spectrum usage detection, and the last one is estimation and modeling of statistical spectrum usage ${ }^{\dagger}$.

Since general wireless system is assumed, we use energy detector (ED) [14] for the spectrum measurement. Specifically, ED does not need any specific information about observed signal, such as modulation scheme. Welch FFT-based ED is an effective method for achieving proper spectrum usage detection [15], [16]. Welch FFT consists of three steps: segmentation of data sequence with a specific segment size, calculation of multiple power spectra and averaging of the power spectra. The segment size determines how many segments the averaging is performed and more averaging provides better usage detection performance but poorer frequency resolution at the same time. Therefore, proper segment size for Welch FFT is important as there is a trade-off between the detection performance and frequency resolution in terms of segment size. Accurate spectrum usage detection and high frequency resolution are required for accurate DC estimation and accurate recognition of bandwidth of spectrum occupied by PUs, i.e., accurate recognition of the vacant spectrum, which is usually denoted by white space (WS), respectively [16].

For the issue in terms of segment size, we formulated the optimum segment size in terms of a criterion which is determined by white space detection ratio (WSDR) performance with a constraint determined by sensitivity required for spectrum usage detection [16]. WSDR is defined by the ratio of estimated WS and actual WS in time and frequency domains, therefore WSDR $=1$ indicates ideal situation, $\mathrm{WSDR}>1$ indicates overestimation of WS, and WSDR $<1$ indicates underestimation of WS. The sensitivity in the constraint is quantified by root mean square error (RMSE) of DC estimation. In fact, the optimum segment size depends on signal-to-noise ratio (SNR) which makes practical and optimum segment size setting difficult [16].

For this difficulty, we proposed a practical method denoted by Exhaustive search based Segment size Selection (E-SS) to select a proper segment size without SNR information [16]. E-SS exploits a relationship between output of noise floor (NF) estimation based on forward consecutive mean excision (FCME) algorithm and the segment size for proper segment size setting. E-SS uses NF estimation outputs for all possible segment sizes in Welch FFT, therefore it requires relatively high computational complexity.

In this paper, we propose a segment size selection method called Limited search based Segment size Selection (L-SS) and it also uses NF estimate via FCME algorithm. L-SS can achieve both reasonable WSDR performance and low computational complexity since it searches the proper

${ }^{\dagger}$ Time-frequency conversion and spectrum usage detection in SAS and spectrum sensing in DSA system are similar in that both decide spectrum occupancy, i.e., whether PU is active or not. However, SAS exploits spectrum occupancy result for estimation of statistical spectrum usage information, but DSA system exploits it for other processes in DSA such as spectrum management, channel selection. segment size while limiting the searchable segment sizes.

Numerical results show that E-SS and L-SS has comparable performances in terms of RMSE of DC estimate and WSDR, while computational complexity of L-SS has sufficiently lower than that of E-SS.

The remainder of the paper is organized as follows. The system model used in this paper is presented in Sect. 2. In Sect. 3, we introduce the Welch FFT segment size design criterion leading an optimum segment size by considering the trade-off between detection performance and frequency resolution. In Sect. 4, we introduce related works regarding segment size selection. Specifically, we present the relationship between SNR, segment size and NF estimate, and the conventional segment size selection method, i.e., E-SS. After that, we propose L-SS in Sect. 5. Performance evaluation based on computer simulation is presented in Sect. 6 . Finally, conclusions are presented in Sect. 7.

\section{System Model}

The block diagram of the signal processing used for spectrum usage measurement is shown in Fig. 1. The process consists of several components: Welch FFT with memory [17], NF estimation consisting of tentative NF estimation and final NF estimation, threshold setting, spectrum usage detection, segment size selection, and DC estimation.

Configuration of time frames for the spectrum usage measurement is shown in Fig. 2. One consecutive spectrum usage measurement consists of $M_{\mathrm{s}}$ super frames and each super frame consists of $M$ time frames. It is desirable to have sufficiently long measurement duration so that multiple primary user signals can be observed for accurately estimating DC. However, practically maximum duration is limited by the memory size in an observation equipment (OE) such as spectrum analyzer in SAS. Welch FFT, spectrum usage detection, and segment size selection are performed to obtain signal usage detection results for every time frame. One time frame consists of $N_{\mathrm{s}}$ complex samples of received sig-

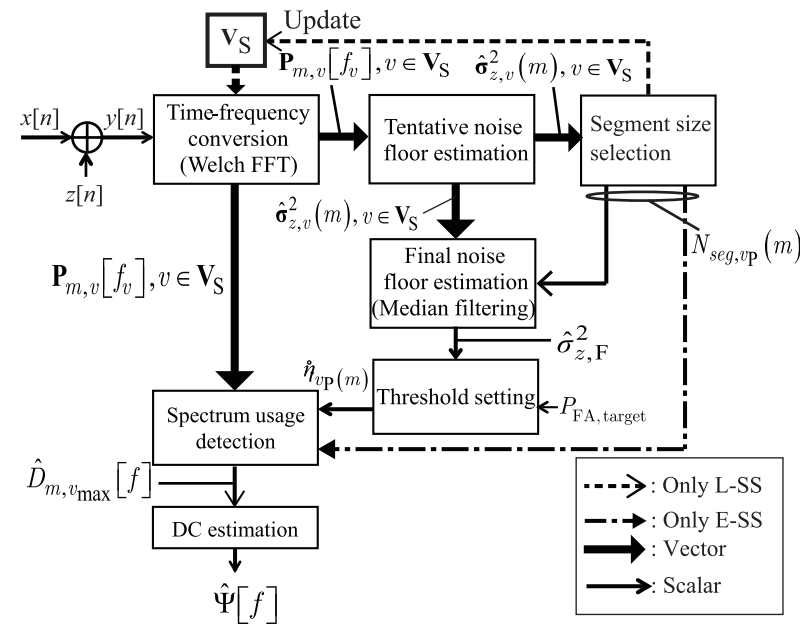

Fig. 1 Block diagram of the spectrum measurement process. 


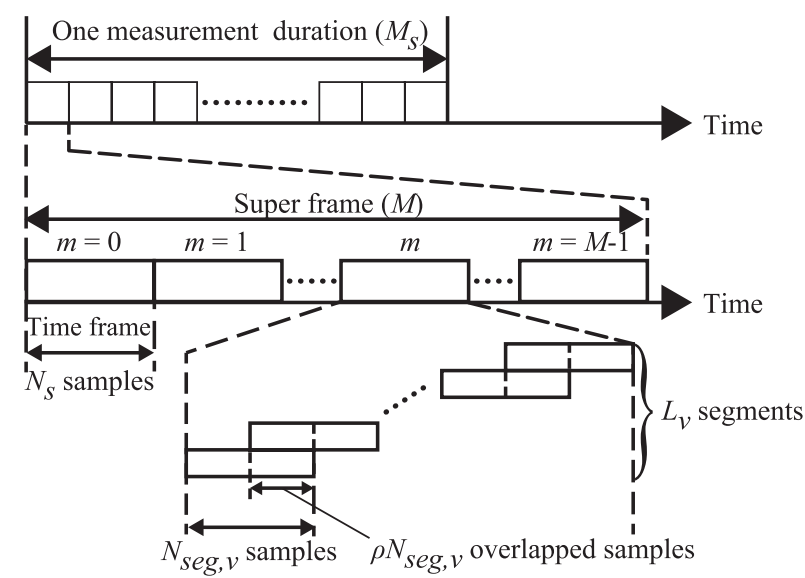

Fig. 2 The configuration of time frames in the measurement process.

nal with sampling rate $f_{\mathrm{s}} \mathrm{Hz}$ and this time frame corresponds to one Welch FFT size. The time frame length determines time resolution which is an important parameter to understand spectrum activity of PUs. The time frame length is set so that required time resolution can be met.

The tentative NF estimation using FCME algorithm [18] is performed in every time frame and the final NF estimation is obtained by median filtering the $M$ tentative NF estimates [19]. The one super frame size $M$ corresponds to median filter length for final NF estimation. NF estimation accuracy can be improved by using a median filter for NF estimation [20]. Optimization of super frame length (median filter length) is out of our scope and we determine the length according to [20]. The rationale for using the median filter for NF estimation is that NF is usually almost static at least a few minutes [20], [21]. The DC estimation is obtained based on $M_{\mathrm{s}} \times M$ signal usage detection results.

SAS observes the wide band spectrum in which several PUs may exist in the observed frequency band and bandwidth is denoted by $W_{\mathrm{M}} \mathrm{Hz}$. We assume that PU's signal bandwidth can vary with time, but the bandwidth is at most $90 \%$ of $W_{\mathrm{M}} \mathrm{Hz}$. The time duration and time interval of PU's signals can be also changed in time, but time resolution of spectrum measurement has to be shorter than the time duration and time interval. The time resolution is determined by the time frame duration. The maximum signal length is assumed to be less than one measurement duration.

Now let us focus on the $m$ th time frame $(m=$ $0,1, \cdots, M-1)$. The $n$th sample of the complex baseband signal $y[n]\left(n=m N_{\mathrm{s}}, m N_{\mathrm{s}}+1, \cdots, m N_{\mathrm{s}}+N_{\mathrm{s}}-1\right)$ in the $m$ th time frame is given by

$$
y[n]= \begin{cases}z[n] & (\mathrm{PU} \text { is not active) } \\ x[n]+z[n] & (\mathrm{PU} \text { is active) }\end{cases}
$$

where $x[n]$ represents the PU signal component and $z[n]$ represents the noise component which follows independent and identically distributed (i.i.d) circular symmetric complex Gaussian distribution with zero mean and variance $\sigma_{z}^{2}$, i.e., $z[n] \sim C N\left(0, \sigma_{z}^{2}\right)$. We assume quasi static fading channel in which channel state is constant during one time frame.
The instantaneous SNR is defined by $\mathrm{SNR}=\sigma_{x}^{2} / \sigma_{z}^{2}$, where $\sigma_{x}^{2}$ and $\sigma_{z}^{2}$ are the instantaneous received PU signal power in $\mathrm{OE}$ and noise power in the observed spectrum, respectively.

In the Welch FFT, $N_{\mathrm{s}}$ samples are segmented into $L_{v}$ segments with an overlap ratio $\rho$. In the rest of the paper, we use $\rho=0.5$ since the signal detection performance at $\rho=0.5$ is appropriate as confirmed in [16]. In $L_{v}, v$ denotes the index number of segment size $\left(v=v_{\min }, v_{\min }+1, \cdots, v_{\max }\right)$ and $V$ denotes the number of all segment sizes as $V=v_{\max }-$ $v_{\min }+1$.

Without loss of generality, $N_{\mathrm{s}}$ and segment size $\left(N_{\mathrm{seg}, v}\right)$ are assumed to be powers of two, i.e., $N_{\mathrm{s}}=2^{v_{\max }}$ and $N_{\mathrm{seg}, v}=$ $2^{v}$, namely $v$ also indicates the exponent of the segment size. In this case,

$$
L_{v}=2 N_{\mathrm{s}} / N_{\mathrm{seg}, v}-1 \text {. }
$$

After the segmentation, normal FFT is performed with respect to each segment and the power spectrum averaged over $L_{v}$ segments is given by [17]

$$
\begin{aligned}
& P_{m, v}\left[f_{v}\right] \\
& =\frac{1}{L_{v}} \sum_{l=0}^{L_{v}-1}\left|\sum_{k=0}^{N_{\text {seg }, v}-1} \frac{\left(w_{v}[k] y\left[k+\frac{l N_{\text {seg }, v}}{2}\right] e^{-j \frac{2 \pi k f_{v}}{N_{\text {seg }, v}}}\right)}{\sqrt{N_{\text {seg }, v}}}\right|^{2},
\end{aligned}
$$

where $f_{v}$ is an index number of the frequency bin $\left(f_{v}=\right.$ $\left.0,1, \cdots, N_{\mathrm{seg}, v}-1\right), m$ is the index number of the time frame and $w_{v}[k]$ is the real-valued window coefficient. The type of window function also affects the detection performance [22]. Here, we use Hamming window because it has been shown that it can achieve slightly better performance compared to other window functions in [19].

Both segment size selection methods, i.e., L-SS and ESS perform Welch FFT with segment sizes specified by a set $\mathbf{V}_{\mathrm{S}}$. In the case of E-SS, the elements in $\mathbf{V}_{\mathrm{S}}$ are all segment sizes $\mathbf{V}_{\mathrm{S}}=\left\{v_{\min }, v_{\min }+1, \cdots, v_{\max }\right\}$ and Welch FFT is performed for each segment in $\mathbf{V}_{S}$ at once, thus, E-SS always performs Welch FFT $V$ times.

On the other hand, in the case of L-SS, $\mathbf{V}_{\mathrm{S}}$ always has only one element and is updated until a proper segment size is found. For this reason, the number of Welch FFT operations can be less than the number in E-SS, i.e, $V$. The reason for the less number of Welch FFT operations in L-SS will be described in Sect. 6.2.

We assume that time resolution $\Delta t=N_{\mathrm{s}} / f_{\mathrm{s}}$ for the duration of a time frame is small enough compared to the minimum continuous signal length such as packet length and the minimum time gap between two consecutive signals, i.e., idle length [5], [23]. On the other hand, the frequency resolution $\Delta f_{v}$ is determined by the segment size as $\Delta f_{v}=f_{\mathrm{s}} / N_{\mathrm{seg}, v}$. We assume the maximum frequency resolution, i.e., $\Delta f_{v_{\max }}=f_{\mathrm{s}} / N_{\mathrm{seg}, v_{\max }}$ is at least narrower than any PU signal bandwidth.

In the $m$ th time frame, segment size selection method (E-SS or L-SS) selects the proper segment size. The background for performing segment size selection every time frame is that in spectrum measurement over wide band including several primary systems, SNR may be changed by 
time frame basis and the optimum segment size depends on SNR. The segment size selection methods, E-SS and L-SS, will be presented in detail in Sects. 4 and 5, respectively. The segment size selected is denoted by $N_{\text {seg, } v_{\mathrm{P}}(m)}$, where $v_{\mathrm{P}}(m)$ denotes the index number of the selected segment size in the $m$ th time frame.

The vector of the tentative NF estimates with the selected segment size is

$$
\hat{\boldsymbol{\sigma}}_{z}^{2}=\left[\hat{\sigma}_{z, v_{\mathrm{P}}(0)}^{2}, \hat{\sigma}_{z, v_{\mathrm{P}}(1)}^{2}, \cdots, \hat{\sigma}_{z, \mathrm{p}_{\mathrm{P}}(M-1)}^{2}\right]^{t},
$$

where the superscript $t$ denotes the vector transpose. The final NF estimate, denoted by $\hat{\sigma}_{z, \mathrm{~F}}^{2}$, is obtained by median filtering the elements in $\hat{\boldsymbol{\sigma}}_{z}^{2}$ [19].

Let $f_{v_{\mathrm{p}}(m)}$ denote the index number of the frequency bin in the $m$ th time frame. Detection result at the $m$ th time frame and the $f_{v_{\mathrm{P}}(m)}$ th frequency bin is obtained by the ED as:

$$
\begin{aligned}
& \hat{D}_{m, v_{\mathrm{P}}(m)}\left[f_{v_{\mathrm{P}}(m)}\right] \\
& = \begin{cases}1 & \text { (if } \left.P_{m, v_{\mathrm{P}}(m)}\left[f_{v_{\mathrm{P}}(m)}\right]>\dot{\eta}_{v_{\mathrm{P}}(m)}\right) \\
0 & \text { (otherwise). }\end{cases}
\end{aligned}
$$

where 1 and 0 respectively correspond to the decisions of occupied spectrum $\left(\mathcal{H}_{1}\right)$ and vacant spectrum $\left(\mathcal{H}_{0}\right)$, and $\dot{\eta}_{v_{\mathrm{p}}(m)}$ indicates the threshold for ED. $\mathcal{H}_{1}$ indicates that PU signal exists in the frequency bin partially or completely and $\mathcal{H}_{0}$ otherwise. In general, the detection performance can be summarized by two probabilities [14]: detection rate $P_{\mathrm{D}}=\operatorname{Pr}\left(P_{m, v_{\mathrm{p}}(m)}\left[f_{v_{\mathrm{p}}(m)}\right]>\dot{\eta}_{v_{\mathrm{P}}(m)} \mid \mathcal{H}_{1}\right)$ and false alarm rate $P_{\mathrm{FA}}=\operatorname{Pr}\left(P_{m, v_{\mathrm{P}}(m)}\left[f_{v_{\mathrm{P}}(m)}\right]>\dot{\eta}_{v_{\mathrm{P}}(m)} \mid \mathcal{H}_{0}\right)$, where $\operatorname{Pr}(x)$ indicates the probability of event $x$. The threshold $\dot{\eta}_{v_{\mathrm{P}}(m)}$ is set based on $\hat{\sigma}_{z \mathrm{~F}}^{2}$, the selected segment size in the $m$ th time frame, and a target false alarm rate $P_{\mathrm{FA} \text {,target }}$. In case of Welch FFT-based ED, proper threshold setting for $P_{\mathrm{FA} \text {,target }}$ or $P_{\mathrm{D}}$ is available [22], [24].

In the spectrum usage detection $\left(\hat{D}_{m, v_{\mathrm{P}}(m)}\left[f_{v_{\mathrm{P}}(m)}\right]\right)$, the number of frequency bins varies every time frame due to the segment size selection. For the DC estimation in each frequency bin, the number of frequency bins in each time frame is unified by the maximum number of frequency bins $N_{\mathrm{s}}=2^{v_{\max }}$. This can be achieved by

$$
\hat{D}_{m, v_{\max }}[f]=\hat{D}_{m, v_{\mathrm{P}}(m)}\left[f_{v_{\mathrm{P}}(m)}\right],
$$

where

$$
\frac{\Delta f_{v_{\mathrm{p}}(m)}}{\Delta f_{v_{\max }}} f_{v_{\mathrm{P}}(m)} \leq f \leq \frac{\Delta f_{v_{\mathrm{p}}(m)}}{\Delta f_{v_{\max }}}\left(f_{v_{\mathrm{P}}(m)}+1\right)-1,
$$

and $\Delta f_{v_{\mathrm{P}}(m)}$ and $\Delta f_{v_{\max }}$ indicate the frequency resolution for segment size $2^{v_{\mathrm{P}}(m)}$, i.e., $\Delta f_{v_{\mathrm{P}}(m)}=f_{\mathrm{s}} / N_{\text {seg, }, v_{\mathrm{P}}(m)}$ and the frequency resolution for largest segment size $N_{\text {seg, } v_{\max }}=N_{\mathrm{s}}$, i.e., $\Delta f_{v_{\max }}=f_{\mathrm{s}} / N_{\mathrm{s}}$, respectively.

DC estimation is performed for each super frame and each frequency bin and the estimated DC at the $f$ th bin is

$$
\hat{\Psi}[f]=\frac{1}{M} \sum_{m=0}^{M-1} \hat{D}_{m, v_{\max }}[f] .
$$

We use $m_{\mathcal{H}_{1}}$-out-of- $M$ model [24] to define the true DC as $\Psi[f]=m_{\mathcal{H}_{1}}[f] / M$, where $m_{\mathcal{H}_{1}}[f]$ denotes the number of $\mathcal{H}_{1}$ hypotheses in the $f$ th frequency bin.

\section{Segment Size Design Criterion}

Larger segment size can achieve high frequency resolution, however it results in reduced signal detection sensitivity due to small $L_{v}$ in (2) and vice versa [15]. To achieve proper segment size, we have formulated in [16] an evaluation criterion for the design of the segment size setting based on WSDR as

$$
\begin{aligned}
v_{\mathrm{OPT}}=\underset{v \in\left\{v_{\min }, v_{\min }+1, \cdots, v_{\max }\right\}}{\arg \min }|1-\mathrm{WSDR}(v)|, \\
\text { s.t. } \operatorname{RMSE}\left(\Psi\left[f_{c}\right]\right) \leq \delta, \\
P_{\mathrm{FA}}=P_{\mathrm{FA}, \text { target }},
\end{aligned}
$$

and the optimum segment size is given by $N_{\text {seg, } v_{\text {OPT }}}=2^{v_{\text {OPT }}}$ and it depends on $\operatorname{SNR}[15]$. $\operatorname{WSDR}(v), \operatorname{RMSE}\left(\Psi\left[f_{\mathrm{c}}\right]\right)$, and $\delta$ denote the WSDR, RMSE in terms of DC estimation at the center frequency $f_{\mathrm{c}}$ for the PU signal, and allowable RMSE for DC estimate, respectively.

In the following subsections, the details of $\operatorname{RMSE}\left(\Psi\left[f_{\mathrm{c}}\right]\right)$ and $\operatorname{WSDR}(v)$ are described.

\subsection{RMSE in Terms of DC Estimate}

The RMSE of DC estimation error in the $m_{\mathcal{H}_{1}}$-out-of-M model is given by [24]

$$
\operatorname{RMSE}\left(\Psi\left[f_{\mathrm{c}}\right]\right)=\sqrt{\mathbb{E}\left[\left(\hat{\Psi}\left[f_{\mathrm{c}}\right]-\Psi\left[f_{\mathrm{c}}\right]\right)^{2}\right]},
$$

where $\mathbb{E}[\cdot]$ denotes expectation, $f_{\mathrm{c}}$ is set to the center of the PU signal in frequency domain, $\Psi\left[f_{\mathrm{c}}\right]$ is the true DC, and $\hat{\Psi}\left[f_{\mathrm{c}}\right]$ is the estimated DC based on the detection results. Typically, $P_{\mathrm{FA} \text {,target }}$ should be set to a small value, such as 0.01 . Therefore, $P_{\mathrm{D}}$ should be high enough to satisfy $\operatorname{RMSE}\left(\Psi\left[f_{\mathrm{c}}\right]\right) \leq \delta$ with small $\delta$. Satisfying the constraint (8) requires accurate enough detection performance in time domain, but frequency resolution is not considered. Note that RMSE in terms of DC can evaluate detection performance, i.e., detection rate $P_{\mathrm{D}}$ and false alarm rate $P_{\mathrm{FA}}$ as well as DC estimation accuracy [16].

\subsection{WSDR}

The criterion WSDR with the constraint in (8) indicates a vacant spectrum detection capability with considering the frequency resolution. WSDR is defined by

$$
\operatorname{WSDR}(v)=\frac{\sum_{f=0}^{\left.N_{\text {seg, } v_{\text {max }}-1}-1-\mathbb{E}[\hat{\Psi}[f]]\right)}}{\sum_{f=0}^{N_{\text {seg, }, v_{\max }-1}-1}(1-\Psi[f])},
$$

where the denominator and numerator indicate true WS and estimated WS, respectively. In this metric, a value closer to one indicates more accurate detection performance. Note that in (10), the effect of frequency resolution is determined by used segment size. 


\section{Related Work of Segment Size Selection [16]}

In this section, at first we will show a relationship between SNR, segment size and NF estimate by the tentative NF estimation with brief description of FCME algorithm. In fact, E-SS and L-SS exploit an aspect of the tentative NF estimate. After that, E-SS algorithm will be described.

\subsection{Relationship between SNR, Segment Size and NF Es- timate}

FCME algorithm [18] used in the tentative NF estimation is an iterative method that attempts to recognize clean power spectrum samples (noise-only samples) which correspond to $\mathcal{H}_{0}$ samples.

More specifically, the process of FCME algorithm is as follows. It first sorts the power spectrum samples in an ascending order. After that, it calculates the mean of the $I$ smallest sorted samples. The NF estimation assumes that at least $I$ smallest sorted samples are noise-only samples (clean samples). In general, $I=\lceil 0.1 N\rceil$, where $\lceil\cdot\rceil$ is the ceiling function and $N$ is the number of frequency bins (segment size), and we also use $I=\lceil 0.1 N\rceil$ throughout the paper according to the related works [19], [20]. By assuming that the calculated mean is correct, the threshold that attains the target false alarm rate such as 0.01 with the calculated mean is obtained based on the distribution of noise power samples, which follows Chi-square distribution with degrees of freedom $2 L_{v}$ [25]. Obviously, the threshold is more than the mean value and the clean samples are updated by adding samples which have value lower than the threshold. Then, the threshold is updated based on the updated clean samples and the target false alarm rate. The updating of clean samples continues as long as new samples are added from the set of non-clean samples (signal plus noise samples) obtained with the latest threshold. Finally, the tentative NF estimate is given by the average power of the estimated clean samples at the final iteration.

From above process of FCME algorithm, NF estimation accuracy is determined by whether the algorithm can accurately divide the sorted power spectrum samples into clean samples and non-clean samples ${ }^{\dagger}$.

Figure 3 shows the average of tentative NF estimates in linear scale as a function of segment size for different SNR, i.e. $-3 \mathrm{~dB}, 0 \mathrm{~dB}$ and $5 \mathrm{~dB}$. The real noise power is set to one. Throughout the paper, the index number of optimum segment size $v_{\mathrm{OPT}}$ is a solution of the optimization problem (8) in which $v$ is the parameter for the optimization problem. The optimum segment size can achieve high enough detection performance while $|1-\operatorname{WSDR}(v)|$ is minimized by a

'If the whole observed band is occupied by PU signals, it may cause overestimation of NF due to non clean samples. However, it may be a rare situation that PUs simultaneously occupy all frequency range since we are typically assuming to use a wide band observation equipment. In addition, median filter can be employed to suppress the effect of the overestimation [20].

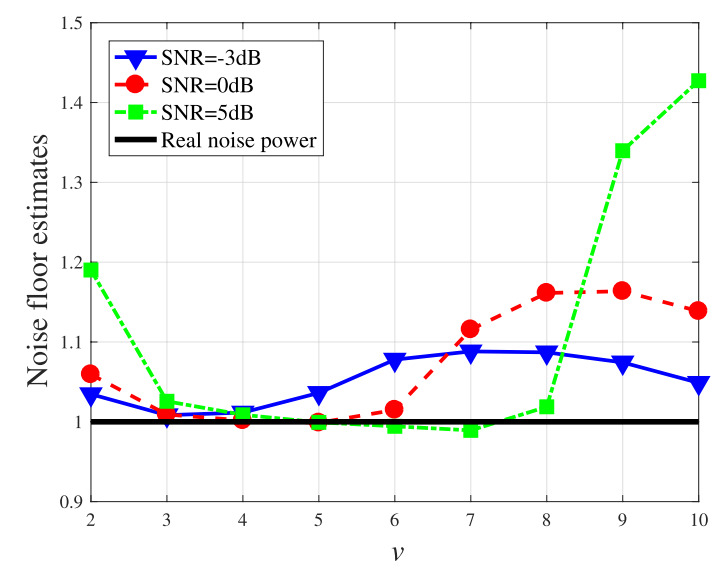

Fig. 3 The relationship between $v$ and tentative NF estimate, SNR $=$ $-3,0,5 \mathrm{~dB}$, the real noise power $\sigma_{z}^{2}=1$.

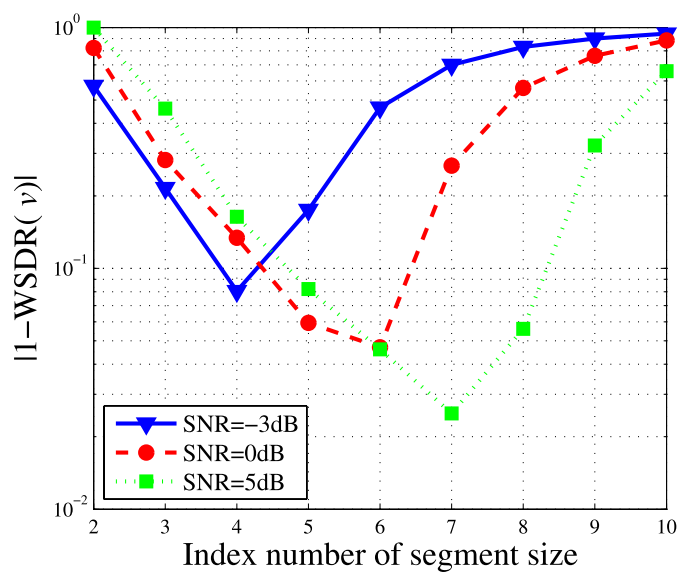

Fig. $4|1-\operatorname{WSDR}(v)|$ as a function of the index number of segment size $v$, $\mathrm{SNR}=-3,0,5 \mathrm{~dB}$.

proper frequency resolution (larger segment size). Figure 4 shows $|1-\operatorname{WSDR}(v)|$ as a function of the index number of segment size $v$ at $\mathrm{SNR}=-3, \mathrm{SNR}=0$ and $\mathrm{SNR}=5 \mathrm{~dB}$. From this figure, the index number of optimum segment size is $v=4$ in $\mathrm{SNR}=-3 \mathrm{~dB}, v=6$ in $\mathrm{SNR}=0 \mathrm{~dB}$ and $v=7$ in $\mathrm{SNR}=5 \mathrm{~dB}$, respectively as $|1-\mathrm{WSDR}(v)|$ is minimized at the index number of optimum segment size while the optimum segment sizes meet the constraint of the optimization problem (8).

FCME algorithm estimates NF by finding a set of clean samples while power spectrum samples are either clean samples or non-clean samples. Specifically, FCME algorithm attempts to find a gap between the clean samples and non-clean samples [26].

In the case of $v=10$, FCME algorithm estimates nonclean samples as clean samples due to no averaging in Welch FFT leading to large fluctuations of power spectrum and difficulty to find the gap [26]. Therefore, it leads to the overestimation of the NF estimate with $v=10$ as shown in Fig. 3. In case of $v=2$, NF estimation accuracy is also poor as shown in Fig. 3. This is reason that some of power spectrum samples (frequency bins) include signal compo- 
nents partially due to the poor frequency resolution and it cannot clearly distinguish between clean samples and nonclean samples. If the segment size is proper $(3 \leq v \leq 8$ in $\mathrm{SNR}=5 \mathrm{~dB}, 3 \leq v \leq 6$ in $\mathrm{SNR}=0 \mathrm{~dB}$ and $3 \leq v \leq 5$ in SNR $=-3 \mathrm{~dB}$ in Fig. 3), NF estimation accuracy is sufficiently high since averaging power spectrum can show the gap accurately.

Both E-SS and L-SS exploit above aspect of the tentative NF estimate, i.e., the slope of tentative NF estimate against the segment size. In fact, the most appropriate segment size is such that it can achieve proper NF estimation with the largest possible segment size to achieve a sufficiently high frequency resolution.

\subsection{Exhaustive Search Based Segment Size Selection: E- SS}

In E-SS, at first Welch FFT and the tentative NF estimation are performed with all segment sizes $N_{\text {seg, } v}$ for each time frame. This provides $V$ tentative NF estimates for the set $\mathbf{V}_{\mathrm{S}}=\left\{v_{\min }, v_{\min }+1, \cdots, v_{\max }\right\}$, i.e., $\left[\hat{\sigma}_{z, v_{\min }}^{2}(m), \cdots, \hat{\sigma}_{z, v_{\max }}^{2}(m)\right]$. The increment of tentative NF estimates between adjacent segment sizes with a positive direction is given by

$$
\Delta \hat{\sigma}_{z, v}^{2}(m)=\hat{\sigma}_{z, v+1}^{2}(m)-\hat{\sigma}_{z, v}^{2}(m) .
$$

Then, the index number of the segment size maximizing the increment is given by

$$
v_{\text {MAX }}(m)=\underset{v}{\arg \max } \Delta \hat{\sigma}_{z, v}^{2}(m) .
$$

The tentative NF estimation with the index number $v_{\operatorname{MAX}}(m)$ can achieve relatively accurate estimation performance. However, it does not necessarily satisfy the RMSE constraint. Therefore, an adjustable integer parameter $\beta$ is employed to achieve the RMSE constraint and the index number of segment size selected by E-SS is

$$
v_{\mathrm{P}}^{(\mathrm{E}-\mathrm{SS})}(m)=v_{\mathrm{MAX}}(m)+\beta .
$$

Thus, the segment size selected by E-SS is $N_{\text {seg, } v_{\mathrm{P}}^{(\mathrm{E}-\mathrm{SS})}(m)}=$ $2^{v_{\mathrm{MAX}}(m)+\beta}$.

The parameter $\beta$ can be set based on RMSE constraint, e.g., $\beta=-1$ can satisfy $\delta \leq 0.05$ as confirmed by Monte Carlo simulations in [16].

\section{Limited Search Based Segment Size Selection: L-SS}

In this section, we present details of L-SS algorithm. A flowchart of the process of L-SS is shown in Fig. 5. It consists of three decisions: decision-1 (D-1), decision-2 (D-2) and decision-3 (D-3) to determine appropriate segment size.

At the $m$ th time frame, the initial value of $\mathbf{V}_{\mathrm{S}}$ is set by an index number of segment size selected in the previous time frame $(m-1): v_{0}(m)=v_{\mathrm{P}}(m-1)$, where subscript 0 indicates the initial value. In the case of $m=0$, we can employ E-SS to select a proper segment size.

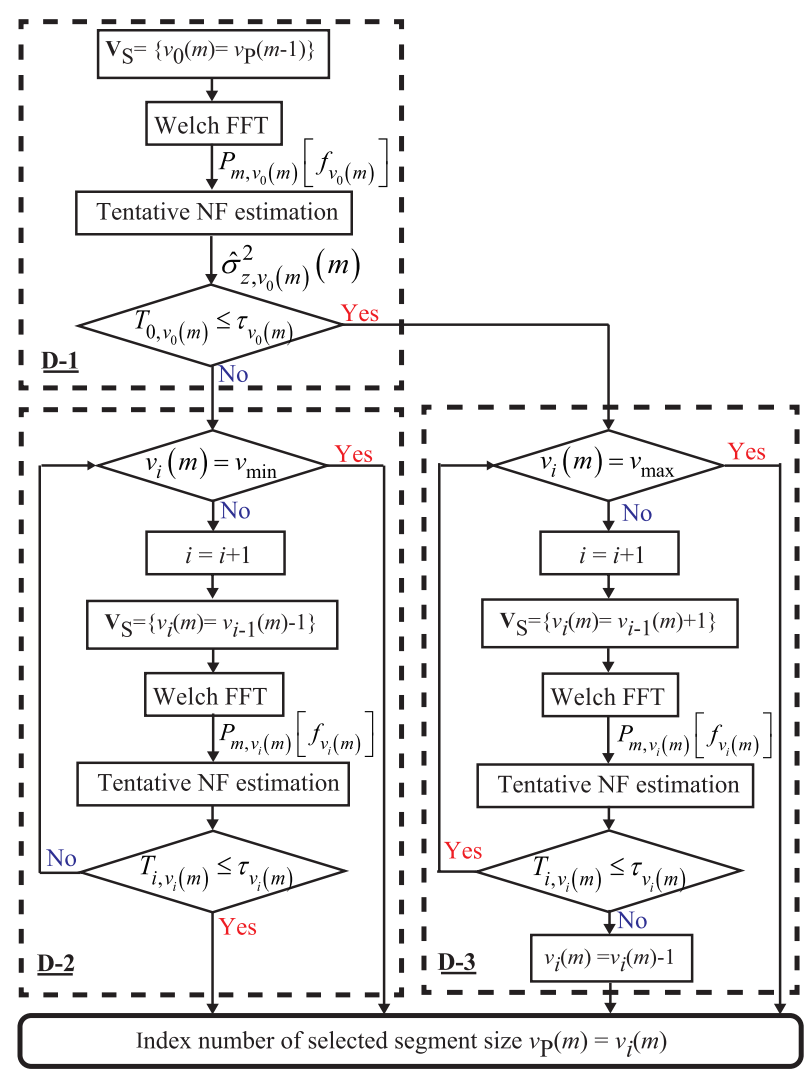

Fig. 5 Flowchart of L-SS

Let $T_{i, v_{i}(m)}$ denote the ratio of the tentative NF estimate $\left(\hat{\sigma}_{z, v_{i}(m)}^{2}(m)\right)$ to the final NF estimate from the previous super frame $\left(\hat{\sigma}_{z, \mathrm{~F}}^{2}\right)$, i.e.,

$$
T_{i, v_{i}(m)}=\frac{\hat{\sigma}_{z, v_{i}(m)}^{2}(m)}{\hat{\sigma}_{z, \mathrm{~F}}^{2}}, i=0,1, \cdots, V-1,
$$

where the subscript $i$ indicates the index number of segment size updating.

Typically, the changing rate of NF is very slow and relatively accurate NF estimate is available by $\hat{\sigma}_{z, \mathrm{~F}}^{2}$. In fact, it has been shown that NF is usually almost static at least a few minutes [20], [21]. Considering this aspect, in the case that $\hat{\sigma}_{z, v_{0}(m)}^{2}(m)$ is significantly larger than $\hat{\sigma}_{z, \mathrm{~F}}^{2}$, i.e., $T_{0, v_{0}(m)}>\tau_{v_{0}(m)}$, it is considered that the current checked segment size $2^{v_{0}(m)}$ is large compared with a proper segment size. This leads to the fact D-1 is "No". Then, the threshold $\tau_{v_{0}(m)}$ is determined by a significance level regarding whether $\hat{\sigma}_{z, v_{0}(m)}^{2}(m)$ is too large compared with $\hat{\sigma}_{z \mathrm{~F}}^{2}$. More detailed description about the threshold setting will be presented at the end of this subsection.

Specifically, in the case that the decision is "No" in D1 , the index number of segment size is updated by $v_{i}(m)=$ $v_{i-1}(m)-1, i=1, \cdots, V-1$, i.e., the set $\mathbf{V}_{\mathbf{S}}=\left\{v_{i-1}(m)-1\right\}$ in D-2. The update is repeated until it achieves an appropriate segment size so that $T_{i, v_{i}(m)} \leq \tau_{v_{i}(m)}$ or that it reaches the smallest segment size $N_{\text {seq, } v_{\min }}$.

On the other hand, the decision "Yes" in D-1 indicates 
that the segment size is not too large and proper NF estimation performance may be achievable. However, there is a possibility that the segment size is too small, for example due to increase of SNR.

In this case, it goes to D-3 and the segment size is updated in the increasing direction until an inappropriate segment size is found or that it reaches the largest segment size $N_{\text {seg, } v_{\max }}$. Once the inappropriate segment size is found by the decision "No" in D-3, the index number of selected segment size is given by $v_{\mathrm{P}}(m)=v_{i}(m)-1$ since the last updated segment size is the most appropriate segment size.

We set $\tau_{v_{i}(m)}$ so that the following probability equals to a given target probability $P_{\mathrm{L}-\mathrm{SS}}$.

$$
\begin{aligned}
P_{\mathrm{L}-\mathrm{SS}} & =\operatorname{Pr}\left(T_{i, v_{i}(m)}>\tau_{v_{i}(m)} \mid \hat{\sigma}_{z, \mathrm{~F}}^{2}=\sigma_{z}^{2}, \mathcal{H}_{0}\right) \\
& =\int_{\tau_{v_{i}(m)}}^{\infty} p\left(T_{i, v_{i}(m)} \mid \hat{\sigma}_{z, \mathrm{~F}}^{2}=\sigma_{z}^{2}, \mathcal{H}_{0}\right) d T_{i, v_{i}(m)},
\end{aligned}
$$

where $p\left(T_{i, v_{i}(m)} \mid \hat{\sigma}_{z, \mathrm{~F}}^{2}=\sigma_{z}^{2}, \mathcal{H}_{0}\right)$ is the conditional probability density function (PDF) of $T_{i, v_{i}(m)}$ under $\hat{\sigma}_{z, \mathrm{~F}}^{2}=\sigma_{z}^{2}$ and $\mathcal{H}_{0}$ and it is obtained via Monte Carlo simulations. $P_{\mathrm{L}-\mathrm{SS}}$ means a significance level regarding whether $\hat{\sigma}_{z, v_{i}(m)}^{2}(m)$ is too large compared with $\hat{\sigma}_{z, \mathrm{~F}}^{2}$, thus whether the current checked segment size is too large compared with a proper segment size.

\section{Numerical Evaluations}

In this section, we will evaluate two segment size selection methods, i.e., L-SS and E-SS. The evaluated metrics are as follows: RMSE in terms of DC, $|1-\operatorname{WSDR}(v)|$ and computational complexity. In addition, we will also evaluate the adaptivity of segment size selection with time, i.e., the behavior of L-SS and E-SS in case that SNR is varied in time of L-EE and E-SS.

We assume that the measurement bandwidth (equivalent to complex sampling rate ) is set to $W_{\mathrm{M}}=f_{\mathrm{s}}=44 \mathrm{MHz}$ and the PU signal bandwidth $W_{\mathrm{S}}=22 \mathrm{MHz}$, such as the bandwidth in IEEE 802.11g WLAN. In addition, the duration and interval of PU signals are constant and the DC $\Psi$ is 0.5 . Specifically, the time duration and the time interval are about $230 \mu \mathrm{sec}$. The time frame size is set to $N_{\mathrm{s}}=1024$ [5], [23] and the time resolution $\Delta t=N_{\mathrm{s}} / f_{\mathrm{s}}$ is $1024 / 44 \times 10^{6} \approx 23 \mu$ sec. $\Delta t$ is short enough compared with the time duration and the time interval. Moreover, we apply $2^{3}$ as the minimum segment size with minimum frequency resolution, $44 \times 10^{6} / 2^{3} \approx 5.5 \mathrm{MHz}$, which is narrow enough compared with the signal bandwidth, $22 \mathrm{MHz}$. Common simulation parameters are summarized in Table 1.

\subsection{RMSE in Terms of DC and $|1-\operatorname{WSDR}(v)|$}

Figure 6 shows $\operatorname{RMSE}\left(\Psi\left[f_{\mathrm{c}}\right]\right)$ as a function of SNR to confirm whether the RMSE constraint is satisfied. In Fig. 7, $|1-\operatorname{WSDR}(v)|$ for every time frame as a function of SNR is shown to confirm the ability to find the WS. $|1-\mathrm{WSDR}(v)|$ indicates the Mean Absolute Error (MAE) of WSDR and
Table 1 Simulation parameters.

\begin{tabular}{|c|c|}
\hline Parameter & Value \\
\hline Number of FFT samples: $N_{\mathrm{s}}$ & $2^{10}$ \\
\hline Segment size: $v$ & $\begin{array}{c}\left\{v_{\min }=3,4,5,6,\right. \\
\left.7,8,9, v_{\max }=10\right\}\end{array}$ \\
\hline Length of median filter: $M$ & 100 \\
\hline Noise Floor $\sigma_{z}^{2}$ & 1 \\
\hline SNR & {$[-410] \mathrm{dB}$} \\
\hline Window type & 0.05 \\
\hline Allowable RMSE: $\delta$ & 0.01 \\
\hline Target false alarm rate: $P_{\mathrm{FA}, \text { target }}$ & 0.5 \\
\hline DC: $\Psi[f], f \in \mathcal{H}_{1}$ & $10^{-3}$ \\
\hline Target probability in L-SS: $P_{\mathrm{L}-\mathrm{SS}}$ & \\
\hline &
\end{tabular}

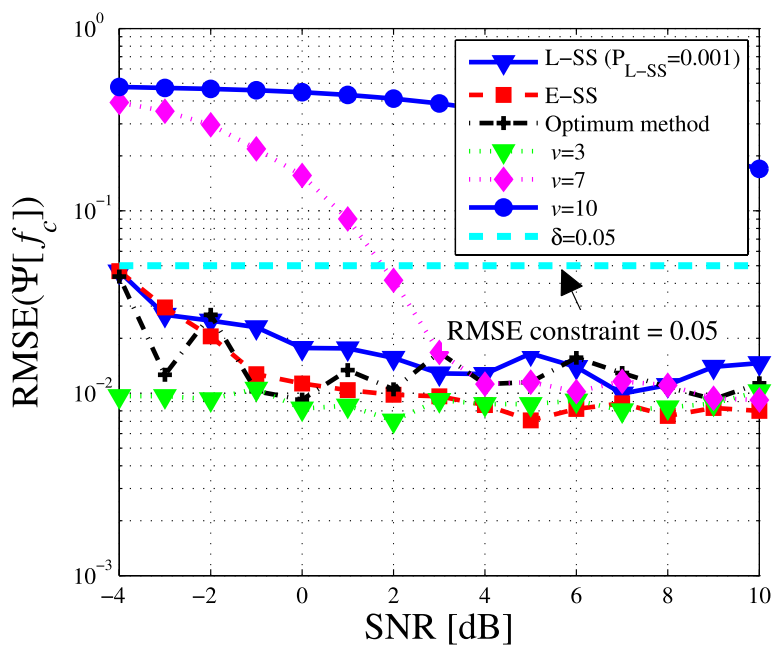

Fig. 6 RMSE $\left(\Psi\left[f_{\mathrm{c}}\right]\right)$ against $\mathrm{SNR}$.

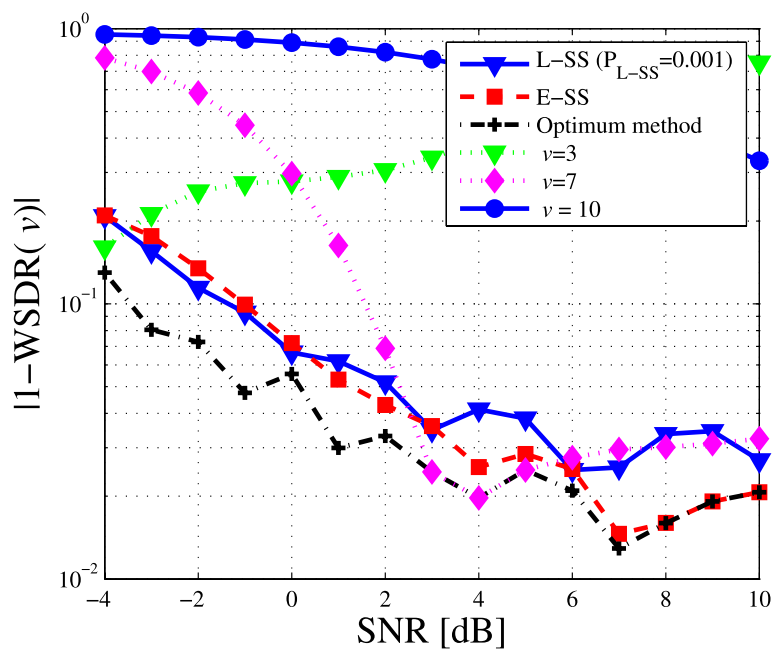

Fig. $7|1-\operatorname{WSDR}(v)|$ in time frames against SNR.

$|1-\mathrm{WSDR}(v)|$ closer to zero indicates more accurate WS detection performance. In the results of Figs. $6\left(\operatorname{RMSE}\left(\Psi\left[f_{\mathrm{c}}\right]\right)\right)$ and $7(|1-\mathrm{WSDR}(v)|)$, five methods are evaluated: the results of L-SS and E-SS, the optimum method where the optimum segment size satisfying (8) is always used, and fixed segment size: $v=3, v=7$ and $v=10$. 
From the results of $v=3, v=7$ and $v=10$ in Figs. 6 and 7 , we can see that using constant segment size cannot achieve proper performance when SNR is changed. When SNR $<2 \mathrm{~dB}$ in Fig. $6, v=7$ is too large to satisfy $\delta=$ 0.05 . In addition, $v=10$ no longer satisfy $\delta=0.05$ at SNR $<10 \mathrm{~dB}$. These indicate the detection performance in low SNR and large segment size is not very good and $v=7$ and $v=10$ are not adequate at SNR $<2 \mathrm{~dB}$ and SNR $<10 \mathrm{~dB}$, respectively. On the other hand, in the case of $v=3$ the RMSE constraint $\delta$ can be satisfied in any SNR. However, Fig. 7 reveals that $|1-\operatorname{WSDR}(v)|$ with $v=3$ is greater than that with $v=7$ at $\mathrm{SNR} \geq 2 \mathrm{~dB}$. This is due to reduced frequency resolution and indicates that the WS cannot be found properly compared with $v=7$, and at least $v=7$ is adequate at $\mathrm{SNR} \geq 2 \mathrm{~dB}$.

In Fig. 6, the optimum method, and the results of ESS and L-SS can always satisfy the RMSE constraint. In addition, the results of E-SS is better than that of L-SS. In Fig. 7, we can confirm that $|1-\operatorname{WSDR}(v)|$ of the optimum method can achieve the best performance in any SNR. From the results of E-SS and L-SS in Fig. 7, we can see E-SS can achieve better $|1-\mathrm{WSDR}(v)|$ performance in any SNR.

We can see that optimum method, E-SS and L-SS have bumpy properties in terms of RMSE. However the results of $v=3$ and $v=7$ have smooth curves. When $2 \mathrm{~dB}<$ $\mathrm{SNR} \leq 5 \mathrm{~dB}$ in Fig. 6 , the index number of the optimum segment size is $v=7$, therefore the RMSE of $v=7$ is equal to the RMSE of the optimum one. However, at $\mathrm{SNR}=6 \mathrm{~dB}$, the index number of the optimum segment size is $v=8$, so the RMSE is slightly increased in a discontinuous manner. Specifically, increasing SNR with fixed segment size leads to a smooth curve, but changing segment size causes the bumpy properties. Obviously, the three methods, i.e., L-SS, E-SS and the optimum method would change the segment size in response to SNR which leads to this behavior.

\subsection{Computational Complexity}

We only evaluate the computational complexity of segment size selection process in L-SS and E-SS. As mentioned in Sect. 4.2 and Sect. 5, E-SS and L-SS consist of Welch FFT and the tentative NF estimation. Thus, the computational complexity of L-SS and E-SS is determined by the computational complexity of Welch FFT and the tentative NF estimation in L-SS and E-SS. Inherently, the computational complexity of L-SS is lower than E-SS since the numbers of executions of Welch FFT and the tentative NF estimation in L-SS are always equal to or less than ones of E-SS. The reason of this aspect is as follows. In E-SS, Welch FFT and the tentative NF estimation are performed for all possible segment sizes. On the other hand, in L-SS, Welch FFT and the tentative NF estimation are performed for a part of them and the details of L-SS are shown in Sect. 5.

We quantitatively evaluate the mean computational time of L-SS and E-SS (Fig. 8). In this evaluation, we use the same simulation parameters as used in Figs. 6 and 7. We can confirm the mean computational time of L-SS is lower

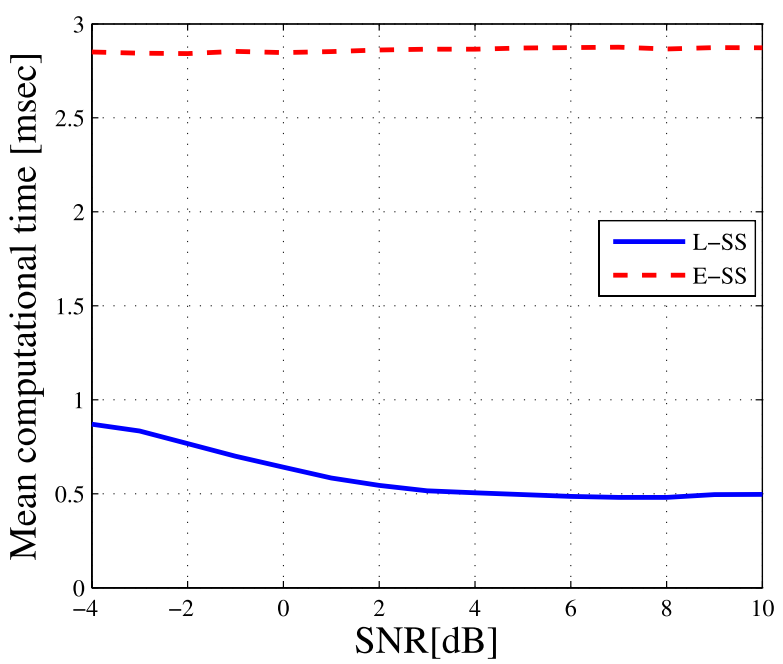

Fig. 8 Computational time of L-SS and E-SS. Computer specifications: Intel Core i7-2600, $3.4 \mathrm{GHz}$, memory size is $8 \mathrm{~GB}$, and the algorithms were implemented in MATLAB.

than that of E-SS.

\subsection{Adaptivity of Segment Size Selection with Time}

Finally, we observe the behavior of L-SS and E-SS in case that SNR is varied in time. Changes of spectrum occupancy state and SNR are summarized in Table 2.

Figure 9 shows ED results in time and frequency domains of L-SS (Fig. 9(a)) and E-SS (Fig. 9(b)), respectively. The spectrum occupancy state and SNR are changed in time in Fig. 9 and the changes are shown in Table 2. For comparison, the results of $v=3$ and $v=10$ where segment sizes $N_{\text {seg }, v}=2^{3}$ and $N_{\text {seg }, v}=2^{10}$ are used during the whole observation, respectively are also shown. The PU signal exists between $f=260$ to $f=770$, where $f$ is the index number of frequency bin.

In case of $v=3$ (Fig. 9(c)), the probability of detection is high at the expense of less frequency resolution. This leads to overestimation in terms of the signal bandwidth. In contrast to the case of $v=3$, high frequency resolution is achieved but detection performance is poor in case of $v=10$ (Fig. 9(d)).

On the other hand, we can see both L-SS and E-SS can achieve enough detection performance and this indicates both methods can adaptively select a proper segment size, and detection performance of L-SS and E-SS are comparable in the results of Fig. 9(a) and (b). One difference is frequency resolution: bandwidth of false alarm in L-SS is less than that in E-SS. This indicates that the selected segment size for E-SS is smaller than that for L-SS. To verify this fact, Figs. 10 and 11 show the index number of segment size selected by L-SS and E-SS, respectively. The optimum segment sizes are also plotted by dashed lines in Figs. 10 and 11. In Figs. 10 and 11, the spectrum occupancy state and SNR are changed in time as shown in Table 2. From these figures, both methods can select the segment size around 
Table 2 Spectrum occupancy pattern and SNR change pattern.

\begin{tabular}{|c|c|c|c|c|c|c|c|c|c|c|}
\hline Index number of time frame & $1-10$ & $11-20$ & $21-30$ & $31-40$ & $41-50$ & $51-60$ & $61-70$ & $71-80$ & $81-90$ & $91-100$ \\
\hline State & $\mathcal{H}_{1}$ & $\mathcal{H}_{0}$ & $\mathcal{H}_{1}$ & $\mathcal{H}_{0}$ & $\mathcal{H}_{1}$ & $\mathcal{H}_{0}$ & $\mathcal{H}_{1}$ & $\mathcal{H}_{0}$ & $\mathcal{H}_{1}$ & $\mathcal{H}_{0}$ \\
\hline SNR [dB] & 5 & - & 15 & - & -3 & - & 9 & - & 1 & - \\
\hline
\end{tabular}

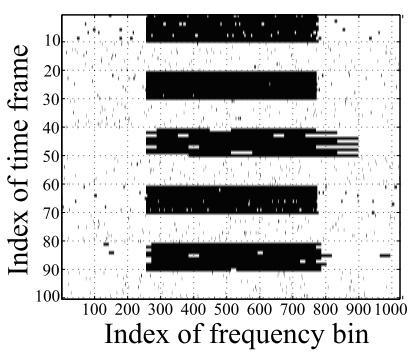

(a) L-SS

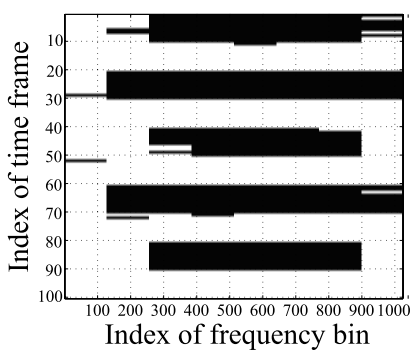

(c) $v=3$

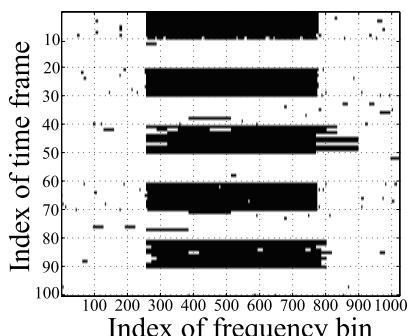

(b) E-SS

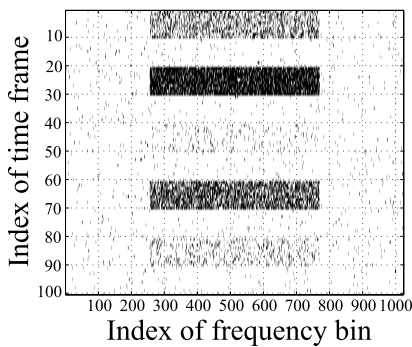

(d) $v=10$
Fig. 9 ED detection results against time frames and frequency bins, (a) L-SS, (b) E-SS, (c) $v=3$, (d) $v=10$.

the optimum segment size, but E-SS selects the smaller segment size than that L-SS selects, especially in high SNR, i.e., $\mathrm{SNR}=15 \mathrm{~dB}$. In both results, we can confirm a biased aspect. Specifically, at a certain time frame, selected segment may be higher (or lower) than the optimum one. The reason of this aspect is that the threshold, $\tau$ (L-SS) and $\beta$ (E-SS) to select a segment size is constant for whole SNR. In fact, the proper $\tau$ and $\beta$ slightly depend on SNR. One important fact is that the maximum error of the segment size selections is one at most. The error is given by the difference between index number of the optimum segment size and index number of segment size selected by L-SS/E-SS.

\section{Conclusion}

We investigated Welch FFT-based ED for FFT-based wide band spectrum measurement in SAS. We aimed to estimate the DC and the WS accurately via Welch FFT-based ED as it is important for SAS to provide accurate statistical information, e.g., DC to SUs. In Welch FFT-based ED, time resolution, frequency resolution and spectrum usage detection performance determine the WS detection performance in the time and frequency domains. The optimum segment size regarding White Space Detection Ratio (WSDR) with the constraint on RMSE in terms of DC depends on the SNR which is an unknown parameter in practice. Therefore, we previously proposed a segment size selection method denoted by Exhaustive search based Segment size Selection (E-SS) to select a proper segment size without SNR

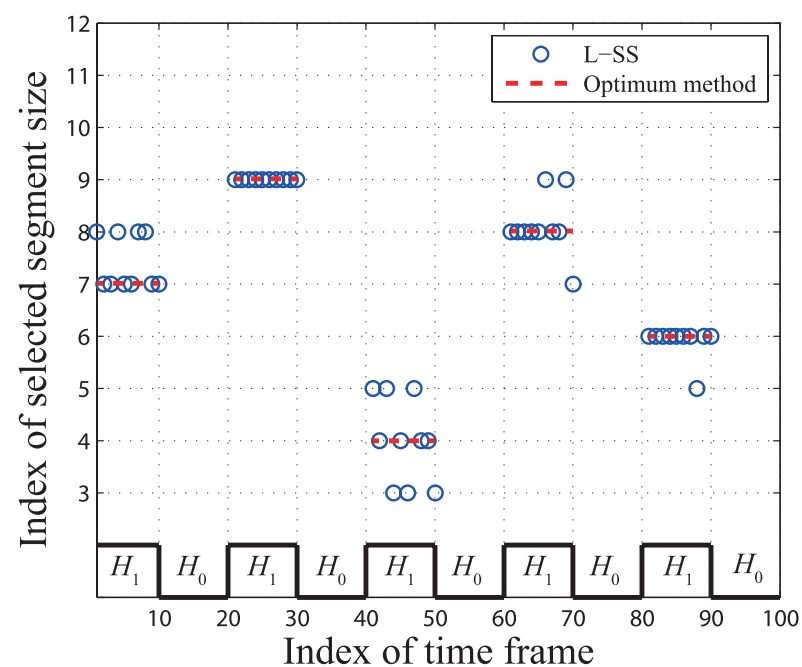

Fig. 10 Index number of selected segment size for L-SS against time frames.

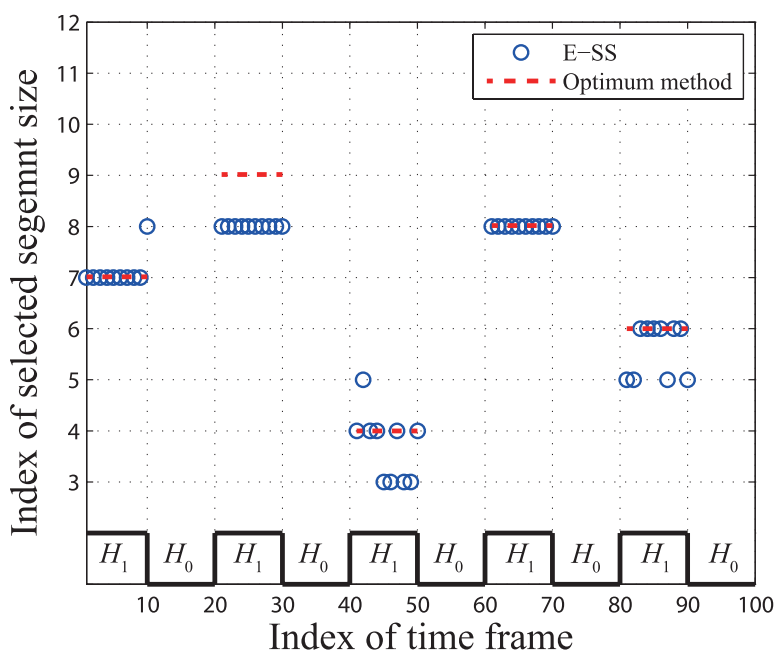

Fig. 11 Index number of selected segment size for E-SS against time frames.

information, but this method requires relatively high computational complexity. For this issue, we proposed Limited search based Segment size Selection (L-SS), which attempts to select the proper segment size with high-computational efficiency. Numerical evaluations showed that L-SS can match the performance of E-SS in terms of MAE of WSDR, i.e., $|1-\operatorname{WSDR}(v)|$ with sufficient DC estimation accuracy. On the other hand, we showed L-SS has much lower computational complexity than E-SS.

\section{Acknowledgements}

This research and development work was supported by 
the MIC/SCOPE \#165003006, and JSPS KAKENHI Grant Numbers JP15K06053, JP15KK0200.

\section{References}

[1] H. Iwata, K. Umebayashi, S. Tiiro, J.J. Lehtomäki, and Y. Suzuki, "A study on welch FFT segment size selection method for spectrum awareness," Proc. IWSS at WCNC, pp.252-257, April 2016.

[2] I.F. Akyildiz, W.Y. Lee, M.C. Vuran, and S. Mohanty, "NeXt generation/dynamic spectrum access/cognitive radio wireless networks: A survey," Computer Networks Journal (Elsevier), vol.50, no.13, pp.2127-2159, Sept. 2006.

[3] T. Yücek and H. Arslan, "A survey of spectrum sensing algorithms for cognitive radio applications," IEEE Commun. Surveys Tuts. vol.11, no.1, pp.116-130, March 2009.

[4] K. Umebayashi, S. Tiiro, and J.J. Lehtomäki, "Development of a measurement system for spectrum awareness," Proc. 5GU, pp.234 239, Nov. 2014.

[5] K. Umebayashi, K. Moriwaki, R. Mizuchi, H. Iwata, S. Tiiro, J.J. Lehtomäki, M. López-Benítez, and Y. Suzuki, "Simple primary user signal area estimation for spectrum measurement," IEICE Trans. Commun., vol.E99-B, no.2, pp.523-532, Feb. 2016.

[6] T. Fujii and K. Umebayashi, "Smart spectrum for future wireless world," IEICE Trans. Commun., vol.E100-B, no.9, pp.1661-1673, Sept. 2017.

[7] N. Wang, Y. Gao, and X. Zhang, "Adaptive spectrum sensing algorithm under different primary user utilizations," IEEE Commun. Lett., vol.17, no.9, pp.1838-1841, Sept. 2013.

[8] T. Nguyen, B.L. Mark, and Y. Ephraim, "Spectrum sensing using a hidden bivariate Markov model," IEEE Trans. Wireless Commun., vol.12, no.9, pp.4582-4591, Sept. 2013.

[9] K. Umebayashi, K. Hayashi, and J.J. Lehtomäki, "Threshold-setting for spectrum sensing based on statistical information," IEEE Commun. Lett., vol.21, no.7, pp.1585-1588, April 2017.

[10] M. Wellens, J. Riihijarvi, and P. Mahonen, "Evaluation of adaptive mac-layer sensing in realistic spectrum occupancy scenarios," 2010 IEEE Symposium on New Frontiers in Dynamic Spectrum (DySPAN), pp.1-12, April 2010.

[11] K. Umebayashi, Y. Suzuki, and J. Lehtomäki, "Dynamic selection of CWmin in cognitive radio networks for protecting IEEE 802.11 primary users," Proc. CROWNCOM, pp.266-270, June 2011.

[12] Y. Xu, A. Anpalagan, Q. Wu, L. Shen, Z. Gao, and J. Wang, "Decision-theoretic distributed channel selection for opportunistic spectrum access: Strategies, challenges and solutions," IEEE Commun. Surveys Tuts., vol.15, no.4, pp.1689-1713, Fourth 2013.

[13] M. Lopez-Benitez and F. Casadevall, "Empirical time-dimension model of spectrum use based on a discrete-time Markov chain with deterministic and stochastic duty cycle models," IEEE Trans. Veh. Technol., vol.60, no.6, pp.2519-2533, July 2011.

[14] H. Urkowitz, "Energy detection of unknown deterministic signals," Proc. IEEE, vol.55, no.4, pp.523-531, April 1967.

[15] H. Iwata, K. Umebayashi, S. Tiiro, Y. Suzuki, and J.J. Lehtomäki, "Optimum welch FFT segment size for duty cycle estimation in spectrum awareness system," Proc. IWSS at WCNC, pp.253-258, March 2015.

[16] H. Iwata, K. Umebayashi, S. Tiiro, J.J. Lehtomäki, M. LópezBenítez, and Y. Suzuki, "Welch FFT segment size selection method for spectrum awareness system," IEICE Trans. Commun., vol.E99B, no.8, pp.1813-1823, Aug. 2016.

[17] P.D. Welch, "The use of fast Fourier transform for the estimation of power spectra: A method based on time averaging over short, modified periodograms," IEEE Trans. Audio Electroacoust., vol.15, no.2, pp.70-73, Jun. 1967.

[18] H. Saarnisaari, P. Henttu, and M. Juntti, "Iterative multidimensional impulse detectors for communications based on the classical diagnostic methods," IEEE Trans. Commun., vol.53, pp.395-398, March
2005

[19] J. Lehtomäki, R. Vuohtoniemi, and K. Umebayashi, "On the measurement of duty cycle and channel occupancy rate," IEEE J. Sel. Areas Commun., vol.31, no.11, pp.2555-2565, Nov. 2013.

[20] J.J. Lehtomäki, R. Vuohtoniemi, K. Umebayashi, and J.P. Mäkelä, "Energy detection based estimation of channel occupancy rate with adaptive noise estimation," IEICE Trans. Commun., vol.E95-B, no.4, pp.1076-1084, April 2012.

[21] D. Torrieri, "The radiometer and its practical implementation," Proc. MILCOM, pp.304-310, IEEE, Nov. 2010.

[22] S. Wang, F. Patenaude, and R.J. Inkol, "Computation of the normalized detection threshold for the FFT filter bank-based summation CFAR detector," J. Comput., vol.2, no.6, pp.35-48, Aug. 2007.

[23] S. Geirhofer, L. Tong, and B.M. Sadler, "A measurement-based model for dynamic spectrum access in WLAN channels," Proc. MILCOM, pp.1-7, Oct. 2006.

[24] J. Lehtomäki, M. López-Benítez, K. Umebayashi, and M. Juntti, "Improved channel occupancy rate estimation," IEEE Trans. Commun., vol.63, no.3, pp.643-654, March 2015.

[25] S.M. Kay, Fundamentals of Statistical Signal Processing, Prentice Hall PTR, 1998.

[26] K. Umebayashi, R. Takagi, N. Ioroi, Y. Suzuki, and J. Lehtomäki, "Duty cycle and noise floor estimation with welch FFT for spectrum usage measurements," Proc. CROWNCOM, pp.73-78, June 2014.

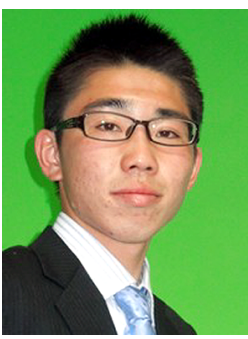

Hiroki Iwata was born in Miyazaki, Japan in 1992. He received his B.E. and M.E. degrees in electrical and electronic engineering from Tokyo University of Agriculture and Technology, Tokyo, Japan, in 2014 and 2016, respectively. Since April 2016, he has been working towards his Ph.D. degree with the Tokyo University of Agriculture and Technology. His research interests are cognitive radio networks, spectrum measurement and wireless communication systems. He is a student member of IEEE.

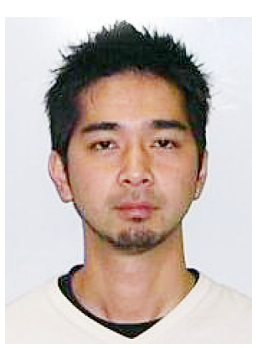

Kenta Umebayashi received LL.B., degree from Ritsumeikan University in 1996, B.E., M.E., and Ph.D. degrees from the Yokohama National University in 1999, 2001, and 2004, respectively. From 2004 to 2006, he was a research scientist at the University of Oulu, Centre for Wireless Communications (CWC). He is currently an assistant professor in the Tokyo University of Agriculture and Technology. In 2010, he was a visiting professor at the University of Oulu. His research interests lie in the areas of signal detection theory and wireless communication systems, cognitive radio. He received the Best Paper Award in IEEE WCNC 2012 for a paper he authored. He is a member of IEEE. 


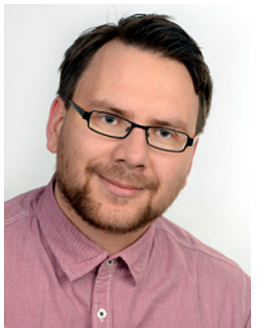

Janne J. Lehtomäki got his doctorate in wireless communications from the University of Oulu in 2005. Currently, he is a senior research fellow at the University of Oulu, Centre for Wireless Communications. He spent the fall 2013 semester at the Georgia Institute of Technology, Atlanta, USA, as a visiting scholar. Currently, he is focusing on communication techniques for networks composed of nanoscale devices. Dr. Lehtomäki has served as a guest associate editor for the IEICE Transactions on Communications Special Section (Feb. 2014) and as a managing guest editor for Nano Communication Networks (Elsevier) Special Issue (Dec. 2015). $\mathrm{He}$ co-authored the paper receiving the Best Paper Award in IEEE WCNC 2012. He is editorial board member of Physical Communication (Elsevier) and was the TPC co-chair for IWSS Workshop at IEEE WCNC 2015 and publicity co-chair for ACM NANOCOM 2015.

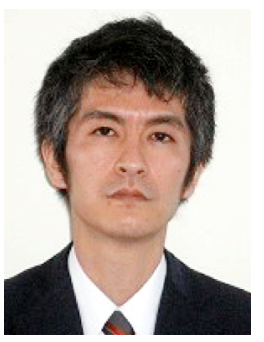

Shusuke Narieda

received the B.E. and M.E. degrees from the University of the Ryukyus and a Dr. Eng. degree from Osaka Prefecture University in 1999, 2001, and 2006, respectively. From 2001 to 2003, he worked at Johokobo Inc. as a software development engineer. From 2006 to 2009, he worked at Yazaki Corp. as an R \& D engineer. He is currently working as an associate professor in the Department of Electrical and Computer Engineering, National Institute of Technology, Akashi College, Japan. His current research interests include distributed signal processing and spectrum sensing techniques for cognitive radio networks, hardware and system design for high-performance wireless communication system, and general signal processing and communication theories. He is a member of the IEEE and IEICE. 